This is the final peer-reviewed accepted manuscript of:

Napolitano, F., F. Tossani, A. Borghetti, and C.A. Nucci. 2018. "Lightning Performance Assessment of Power Distribution Lines by Means of Stratified Sampling Monte Carlo Method." IEEE Transactions on Power Delivery 33 (5): 2571-77.

The final published version is available online at:

https://doi.org/10.1109/TPWRD.2018.2795743

Rights / License:

The terms and conditions for the reuse of this version of the manuscript are specified in the publishing policy. For all terms of use and more information see the publisher's website.

This item was downloaded from IRIS Università di Bologna (https://cris.unibo.it/)

When citing, please refer to the published version. 


\title{
Lightning Performance Assessment of Power Distribution Lines by means of Stratified Sampling Monte Carlo Method
}

\author{
F. Napolitano, Senior Member, IEEE, F. Tossani, Member, IEEE, A. Borghetti, Fellow, IEEE, \\ C. A. Nucci, Fellow, IEEE
}

\begin{abstract}
This paper proposes the application of a recursive stratified sampling technique for improving the assessment of the lightning performance of power distribution lines through the application of the Monte Carlo method. The accuracy of the results is expressed by means of the confidence interval of the estimated annual number of expected overvoltages. The paper shows the results for a single overhead line, with and without surge arresters, and the case of a feeder with laterals. The adoption of the stratified sampling techniques allows for a computational time reduction up to more than $75 \%$ with respect to a standard Monte Carlo procedure. The conceived calculation procedure is applicable to any type of network, regardless of the presence of non-linear devices and avoiding the application of any heuristic rule.
\end{abstract}

Index Terms-Lightning-induced voltages, lightning protection, Monte Carlo method, rare events simulation, stratified sampling, overhead power lines.

\section{INTRODUCTION}

$\mathrm{T}$ HE lightning performance of overhead lines is generally represented by means of curves reporting how many lightning flashovers per year a distribution line may experience as a function of its insulation level [1].

Lightning originated overvoltages in overhead lines are due to both direct strikes and to the coupling between the conductors and the electromagnetic pulse generated by nearby strikes to ground (see, e.g., [2], [3]). For power distribution overhead lines, characterized by limited insulation and height, most of the lightning related flashovers are caused by strikes to the ground or to structures in proximity of the line [1]. As a consequence, the influence of these events on the lightning performance of the line needs to be appropriately assessed to grant the adequate protection.

This paper, therefore, focuses on the calculation of the expected annual number of flashovers due the induced

The Authors are with the Department of Electrical, Electronic and Information Engineering, University of Bologna, 40139 Bologna, Italy.(email: firstname.surname@unibo.it). overvoltages caused by nearby strikes to ground. This calculation has the typical characteristics of rare-event simulation. Indeed, whilst all direct strikes are expected to cause flashovers (unless many surge arresters and shield wires are installed), only a small fraction of nearby strikes to ground are expected to induce voltages larger than the line withstand insulation level.

As described in [4], the standard Monte Carlo (MC) approach for the lightning performance assessment of power distribution lines consists in generating a large number of events, each characterized by different values of lightning current waveshape parameters and by different coordinates of the perspective stroke location (i.e., the stroke location in absence of the line). The values of the lightning current parameters are generated in agreement with the relevant probability distributions available in the literature or provided by lightning location systems (see, e.g., [5], [6]). The coordinates of the stroke location are assumed uniformly distributed within a region around the line wide enough to include all the events that may cause a flashover.

The expected annual numbers of lightning events $F_{p}$ that induce voltages with amplitude larger than a given value of the withstand voltage $W$ is calculated by

$$
F_{p}=\frac{n}{n_{\text {tot }}} A N_{g}
$$

where $n_{t o t}$ is the overall number of randomly-generated events, $n$ is the number of events inducing voltages with peak amplitude larger than $W, A$ is the area that includes the stroke locations of all the dangerous events (i.e., those able to cause voltage peaks exceeding $W$ ), and $N_{\mathrm{g}}$ is the annual ground flash density (in this paper, $N_{\mathrm{g}}$ is assumed equal to $1 \mathrm{flash} / \mathrm{km}^{2} / \mathrm{yr}$ ).

The endpoints of the $95 \%$ confidence interval of the estimate $\hat{p}=n / n_{\text {tot }}$ are $\hat{p} \pm C_{p} \cdot \hat{p}$ where $C_{P}$ is the relative error that can be evaluated as [7]

$$
C_{p}=1.96 \sqrt{\frac{1-p}{n_{\text {tot }} p}} .
$$

under the assumption that the estimator $\hat{p}$ is a normal random variable with mean value $p$ and variance equal to $p(1-p) / n_{\text {tot }}$.

As area $A$ needs to be quite wide and the density of events 
that cause a flashover decreases with the distance from the feeder, $p$ is generally small (rare event). Therefore, in a standard MC approach, $n_{\text {tot }}$ needs to be large so to achieve the desired level of accuracy (i.e., a predefined value of $C_{p}$ ).

In many papers (e.g. [8], [9]), the calculation of the lightning induced overvoltages is performed by using simplified formulas in order to reduce the computational effort. However, as the accurate appraisal of the induced voltages can be achieved only by a time-domain electromagnetic transient simulation (in this paper performed by using the LIOV-EMTP code described in [2], [10], [11] and validated by the comparison of the results with several experimental results [12], [13]), the lightning performance of distribution networks by means of standard MC simulations involves a significant amount of computational resources.

Several recent papers have dealt with the lightning performance assessment by means of electromagnetic transients simulations (e.g. [14]-[16]). Nearby strikes to ground are often referred as indirect strokes in the relevant literature; this term is also used to indicate other indirect lightning events that are not considered in this paper, such as side flashes and the line interaction with a ground current (e.g., [17],[18]). In [19] a method to reduce the computational effort is presented. It is based on the application of the so-called Mean Square Pure Error (MSPE) algorithm to determine the optimal number of Monte Carlo extractions and on a 3D interpolation that bypasses the necessity of the time-domain simulation of each MC event.

In order to reduce the number of LIOV-EMTP simulations, in [20] a heuristic technique has been proposed for the case of an unprotected network, which has been recently adapted in [21] to the case of distribution networks with surge arresters. The heuristic technique is conceived to avoid the time-domain computation of events expected to be less harmful than the previously calculated ones. This requires to fix a priori conditions so to discard the upcoming events which, in general, have characteristics (e.g. amplitude) that depend on the particular network configuration, especially in presence of non-linearities [22].

An improved estimation accuracy can be achieved by two approaches: by increasing the number of replications or by reducing the variance of the estimator. A typical variance reduction technique adopted in $\mathrm{MC}$ methods is the stratified sampling (e.g., [7], [23]). According to this technique, the domain is decomposed into disjoint strata and the MC procedure is applied to each stratum separately.

This paper shows the application of a recursive stratified sampling that consists in the decomposition of area $A$ in subdomains. The number of events generated in each subdomain is proportional to the variance of the local estimator that is recursively updated. Therefore, the larger the distance to the lines or the shorter the distance to surge arresters, the lower the number of events in the specific subdomain. The procedure is conceived to be applicable to any type of network, regardless of the presence of non-linear devices and avoiding any heuristic rule.

The paper is structured as follows. Section II describes the stratified sampling procedure. Section III applies the procedure to a straight-line configuration without and with surge arresters (SAs). Section IV is devoted to the analysis of a feeder configuration with one main and laterals and to the comparison with the heuristic techniques proposed in [20]. Section $\mathrm{V}$ concludes the paper.

\section{STRATIFIED SAMPLING}

Let us define $X$ as a random variable such that $X_{k}=1$ if MC event $k$ causes an overvoltage greater than $W, 0$ if not. As in standard MC methods, the probability $p$ of observing an overvoltage greater than $W$ is estimated by

$$
\hat{p}=\frac{n}{n_{\text {tot }}}=\frac{1}{n_{\text {tot }}} \sum_{k=1}^{n_{\text {tot }}} X_{k}
$$

and the perspective stroke locations of the events in the absence of the power lines and other structures are assumed to be uniformly distributed over area $A$.

As already mentioned, for the application of stratified sampling, total area $A$ is divided in $m$ subdomains and probability $p$ is estimated by

$$
\hat{\vartheta}=\sum_{j=1}^{m} \frac{a_{j}}{A}\left(\frac{1}{N_{j}} \sum_{k=1}^{N_{j}} X_{j k}\right)
$$

where $a_{j}$ is the area of subdomain $j, N_{j}$ is the number of $\mathrm{MC}$ events allocated in subdomain $j$ such that $\sum_{j=1}^{m} N_{j}=n_{t o t}$, and $X_{j k}$ is the $k$-th observation of $X$ in domain $j$. As shown in [23], the variance of estimator $\hat{\vartheta}$ is given by

$$
\operatorname{Var}(\hat{\vartheta})=\sum_{j=1}^{m}\left(\frac{a_{j}}{A}\right)^{2} \frac{\sigma_{j}^{2}}{N_{j}}
$$

where $\sigma_{j}^{2}$ is conditional variance of $X$ in domain $j$. $\operatorname{Var}(\hat{\vartheta})$ is always smaller or equal to $\operatorname{Var}(X) / n_{\text {tot }}$.

Since the values of $\sigma_{j}^{2}$ are not known a priori, they are initially estimated by a certain number of pilot runs, i.e. by the simulation of some MC events. For this purpose, the same number $N_{j}^{s}$ of starting events is generated in each sub-domain, with $N_{j}^{s}$ chosen large enough to estimate $\sigma_{j}^{2}$ even with a very small probability $p$, e.g. in presence of SAs.

In order to obtain a near-random sample distribution for both the parameters of the lightning current waveshape and the stroke location with a limited $N_{j}^{s}$, the starting events in each subdomain $j$ are generated by using the Latin hypercube sampling (LHS) [24]. The use of LHS allows an improved accuracy on the initial estimation of $\sigma_{j}^{2}$ with respect to the usual random sampling.

The endpoints of the $95 \%$ confidence interval of the estimate $\hat{\vartheta}$ are $\hat{\vartheta} \pm C_{\vartheta} \cdot \hat{\vartheta}$ where $C_{\vartheta}$ is the relative error provided by 


$$
C_{\vartheta}=\frac{1.96}{\hat{\vartheta}} \sqrt{\sum_{j=1}^{m}\left(\frac{a_{j}}{A}\right)^{2} \frac{\sigma_{j}^{2}}{N_{j}}} .
$$

Starting from the initial value of $\sigma_{j}^{2}$, the procedure adds new MC events until $C_{\vartheta}$ becomes lower than the desired estimation error.

Each new MC event is allocated in the area $A$ according to a weighted uniform distribution with different weights for each subdomain. The weight of each subdomain $j$ is proportional to the corresponding conditional variance $\sigma_{j}^{2}$ and the sum of the weights of all the $m$ subdomains is equal to 1 . Indeed, the minimum variance value given by (5) is obtained when the events are allocated proportionally to the conditional variance $\sigma_{j}^{2}$ of each subdomain, provided that all subdomains have the same area [23].

If a too small $N_{j}^{s}$ is adopted, a null value can be obtained in the first estimate of $\sigma_{j}^{2}$, in this case the initial guess of the weight of the subdomain can be set according to the mean values of the variances of the adjacent subdomains.

The values of $\sigma_{j}^{2}$ are updated recursively after each MC run, so that their estimation is progressively improved as the number of simulated events increases.

\section{SINGLE LINE}

In the simulations of this paper a linearly rising current with flat top is assumed for the representation of the channel base lightning-current waveform, with peak amplitude $I_{p}$ and equivalent front time $t_{f}$ (an analysis of influence of the use of different waveforms is provided in [25]). The return-stroke propagation speed is set to $1.5 \times 10^{8} \mathrm{~m} / \mathrm{s}$.

The lightning performance is evaluated for overhead lines above a soil with conductivity equal to $0.001 \mathrm{~S} / \mathrm{m}$ and relative permittivity equal to 10 .

Each MC event is characterized by four parameters: $I_{p}, t_{f}$, and the coordinates of the perspective stroke location. Parameters $I_{p}, t_{f}$ are assumed to follow the log-normal probability distributions adopted by Cigré for negative downward first strokes [26]. Each lightning event is classified as either a direct strike to the line or as a nearby strike to ground by using the electro-geometric model suggested in [1].

Since all the direct events in general cause a flashover, their inclusion in the $\mathrm{MC}$ procedure significantly reduces the confidence interval of the obtained results even for a limited number of MC runs. Therefore, for an accurate evaluation of the lightning performance, the annual number of flashovers due to direct strikes and to nearby strike to ground should be evaluated separately. As already mentioned, this paper focuses on the evaluation of the overvoltages due to nearby strikes to ground only.

In this section, the proposed recursive stratified sampling procedure is applied to the case of a single-conductor straight line, assuming a withstand voltage value $W$ equal to $150 \mathrm{kV}$. The line is $2-\mathrm{km}$ long, $10-\mathrm{m}$ high and is matched at both terminations with the matrix of surge impedances to render more straightforward the interpretation of the results.

In Fig. 1, the top view of one half of the considered area $A$ is reported (the line is located on the $x$-axis and the distribution of the stroke locations is obviously symmetric with respect to the line). The figure shows also the stroke locations of all the simulated events, i.e. the initial pilot events and those generated by the stratified sampling procedure. The red dots represent direct strikes to the line whilst the nearby strikes to ground are indicated in blue. For the considered case, the semi-area is a $2 \times 2 \mathrm{~km}$ square, divided in $m=400$ subdomains of area $0.1 \times 0.1 \mathrm{~km}$.

The number of pilot events simulated before starting the stratification procedure is 5,200. The stratified sampling calculation is stopped when relative error $C_{\vartheta}$ reaches the same value of $C_{p}$ calculated with the standard MC method with 100,000 events for $W=150 \mathrm{kV}$.

The total number of events generated by the stratified sampling procedure are 3,464 direct strikes and 23,093 nearby strikes, as reported in Table I. Since only the evaluation of the voltages induced by nearby strikes needs time domain LIOVEMTP simulations, the computational time reduction indicated in Table I is estimated as $100\left(n_{\text {ind }, p}-n_{\text {ind }, \vartheta}\right) / n_{\text {ind, }, p}$, where $n_{\text {ind }, \vartheta}$ is the number of events required by the stratified sampling and $n_{\text {ind, } p}$ is the corresponding number calculated in the standard MC.

As shown by Fig. 1, the procedure allocates the majority of the events in the subdomains closest to the line. A very few events are allocated farther than $1.2 \mathrm{~km}$ from the line, since the initial variance in those subdomains is null.

In order to limit the computational time of the standard MC procedure, the smallest area $A$ that includes all the dangerous events is to be chosen, as discussed in [4]. The capability of the stratified sampling to recursively allocate the events in the subdomains with the largest variance value reduces the importance of an accurate choice of the smallest area $A$.

Fig. 1 also shows that fewer events are allocated near the matched terminations with respect to the subdomains close to the internal part of the line, due to the risers effect that reduce the induced overvoltages [27]. 


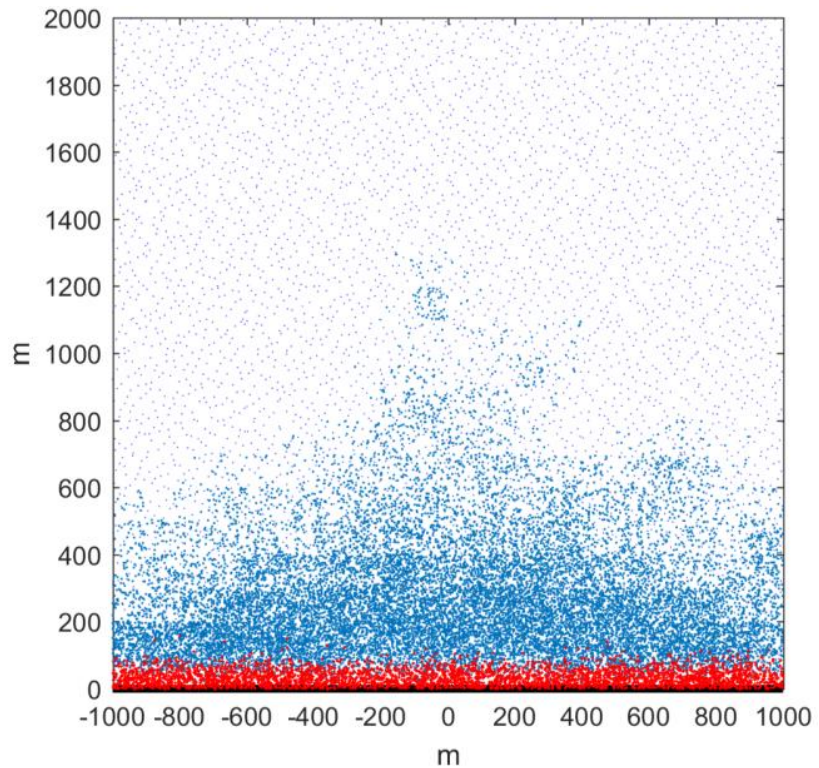

Fig. 1. Position of the events generated by the stratified sampling procedure for the case of an unprotected line (direct strikes in red, nearby strikes to ground in blue).

TABLE I. COMPARISON BETWEEN NUMBER OF DIRECT AND NEARBY STRIKES IN THE STANDARD MC AND STRATIFIED SAMPLING. COMPUTATIONAL TIME REDUCTION DUE TO STRATIFIED SAMPLING.

\begin{tabular}{c|c|c|c|c}
\hline \hline & Relative & Direct strikes & Nearby strikes & Time \\
\cline { 3 - 5 } & error \% & Standard / Stratified & Standard / Stratified & saved \% \\
\hline Unprotected & 2.4 & $3,103 / 3,464$ & $96,897 / 23,093$ & 76 \\
\hline $\begin{array}{c}\text { With SAs } \\
d=500 \mathrm{~m}\end{array}$ & 2.8 & $12,166 / 12,095$ & $187,834 / 42,455$ & 77 \\
\hline $\begin{array}{c}\text { With SAs } \\
d=200 \mathrm{~m}\end{array}$ & 10.7 & $24,665 / 18,687$ & $175,335 / 50,163$ & 71 \\
\hline \hline
\end{tabular}

The above described assessment has been repeated for the case of a line protected with SAs. Two cases are considered with SAs placed every $d=500 \mathrm{~m}$ and $d=200 \mathrm{~m}$, respectively, starting from the line terminations. The line terminations are open. The voltage-current characteristic of the considered SAs is the same used in [28].

For the case of SAs placed every $d=500 \mathrm{~m}$, the semi-area is a $2 \times 1 \mathrm{~km}$ rectangle, divided in $m=20 \times 10$ subdomains of area $0.1 \times 0.1 \mathrm{~km}$. For the case of $d=200 \mathrm{~m}$, the semi-area is a $2 \times 0.5 \mathrm{~km}$ rectangle, divided in $m=20 \times 20$ subdomains of area $0.1 \times 0.025 \mathrm{~km}$. In both cases, the number of pilot runs before starting the stratified sampling procedure is 20,000 . The stratified sampling procedure is stopped when the relative error reaches that achieved by the standard MC with 200,000 events for $W=150 \mathrm{kV}$.

As shown in Table I, the accurate assessment of the performance of a line with SAs requires a very large value of $n_{t o t}$ since, with respect to the case of an unprotected line, the overvoltage becomes a rarer event. Indeed, even with 200,000 events the standard MC procedure does not reach a relative error below $10 \%$ in the case of SAs installed every $200 \mathrm{~m}$.

Fig. 2 shows the position of the simulated lightning events shown for the case of SAs placed every $500 \mathrm{~m}$. In subdomains close to a SA, the values of $\sigma_{j}^{2}$ are very small; therefore, the procedure tends to allocate few events in such subdomains whilst a large number of events is generated in subdomains between two consecutive SAs, where the values of $\sigma_{j}^{2}$ are higher.

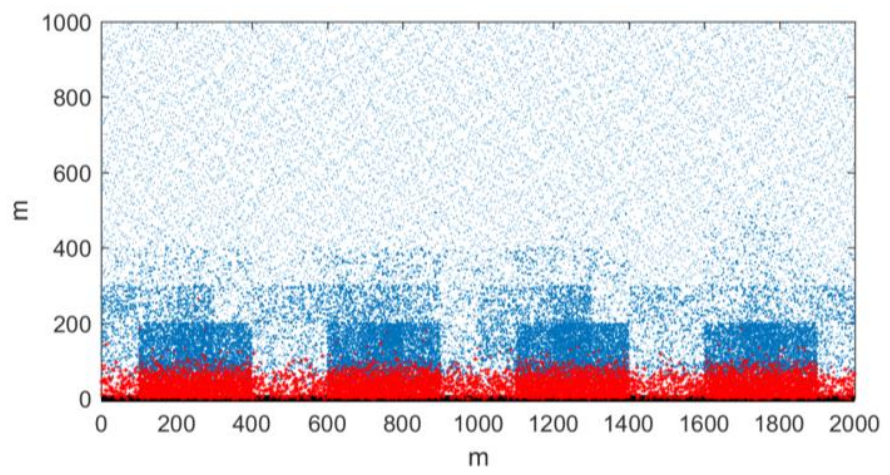

Fig. 2. Position of the events generated by the stratified sampling procedure for the case of a line with consecutive SAs at distance $d=500 \mathrm{~m}$ (direct strikes in red, nearby events to ground in blue).

The presence of SAs considerably reduces the number of dangerous events at distances larger than few hundred meters, as confirmed by Fig. 2. This justifies the choice of a smaller area with respect to the case of an unprotected line. The reduction of the considered area, however, results in an increase of the fraction of direct events, as it can be noticed from the results shown in Fig. 2 and in Table I, particularly for the case with SAs placed at $d=200 \mathrm{~m}$.

Fig. 3 shows the lightning performance for the case of the unprotected line and of the line protected by SAs placed at $d=500 \mathrm{~m}$ and $d=200 \mathrm{~m}$. For each curve, the results are obtained for the same MC event population generated assuming $W=150 \mathrm{kV}$. By using both the standard $\mathrm{MC}$ and the stratified sampling procedure, the $F_{p}$ values at $150 \mathrm{kV}$ are: 0.489 (unprotected), 0.100 (with SAs at $d=500 \mathrm{~m}$ ), 0.003 (with SAs at $d=200 \mathrm{~m}$ ).

Fig. 3 shows that the curves resulting from the application of the stratified sampling are all in close agreement with the results of the standard MC method, for all the withstand values considered in the abscissa.

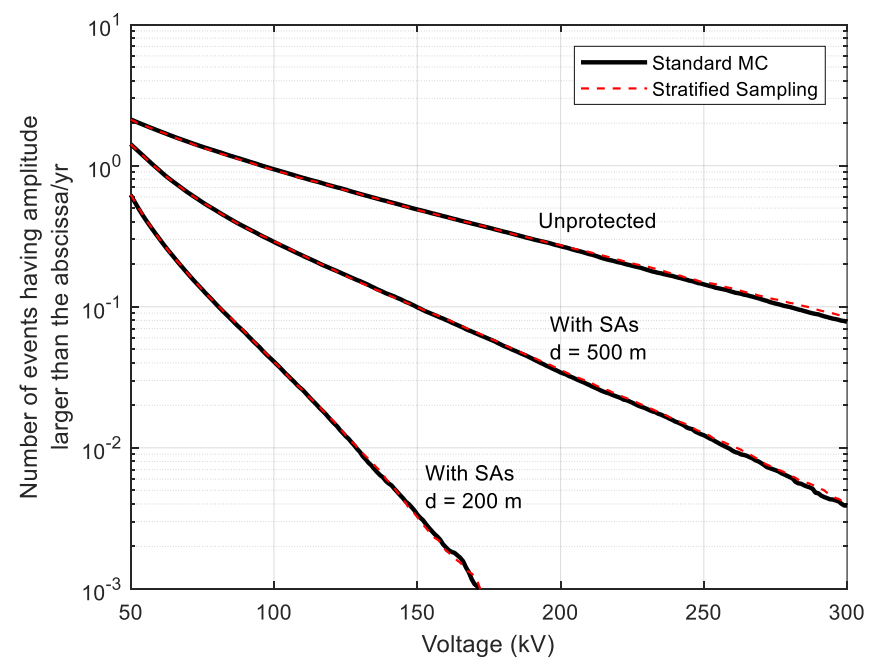

Fig. 3. Comparison of the lightning performance of a line without SAs and with SAs calculated by the standard MC and stratified sampling. 
Fig. 4 compares the values of $C_{P}$ and $C_{\vartheta}$ for different values of $n_{\text {tot }}$ (which accounts for both indirect and direct events). As expected, the value of $C_{\vartheta}$ is always lower or equal than $C_{p}$ for the same value of $n_{\text {tot }}$. Moreover, the figure shows that the use of the stratified sampling allows a fast reduction of the relative error as the number of $\mathrm{MC}$ events increases. As already shown in Table I, the same value of relative error achieved with the standard MC with $n_{t o t}=100,000$ for the unprotected line and $n_{\text {tot }}=200,000$ with SAs are obtained by the stratified sampling with $n_{t o t}=26,557$ (unprotected line), $n_{t o t}=54,550$ (with SAs located every $500 \mathrm{~m}$ ), and $n_{\text {tot }}=68,850$ (with SAs located every $200 \mathrm{~m}$ ).

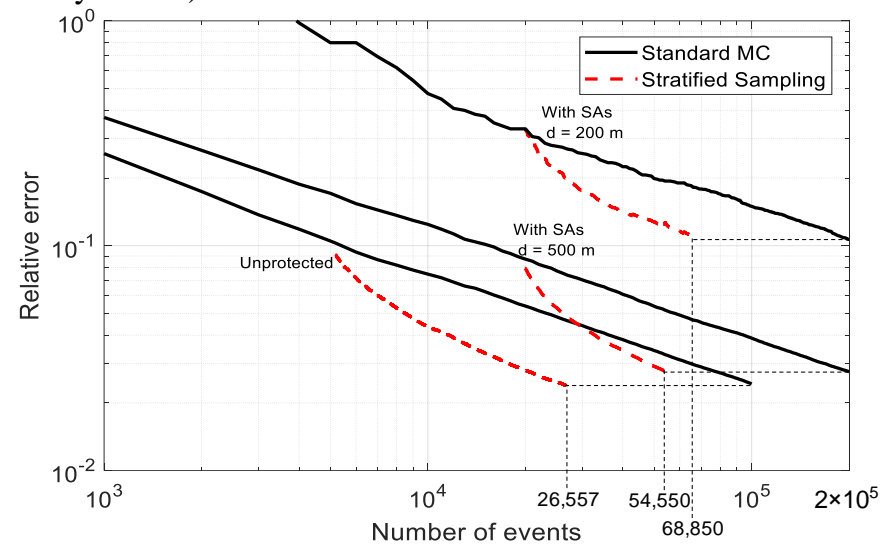

Fig. 4 Comparison of the relative errors calculated for a straight line by the standard MC and stratified sampling as a function of the number of MC events for $W=150 \mathrm{kV}$.

Fig. 5 compares the values of $C_{p}$ and $C_{\vartheta}$ as a function of the considered withstand voltage level for the case without SAs for the case of the unprotected line. As the stratified sampling event population is generated assuming $W=150 \mathrm{kV}, C_{\vartheta}$ is higher than $C_{p}$ for voltages lower than $W$ and its value practically remains constant. This provides a criterion for the choice of $W$, unless the withstand voltage of the line is already defined.

For all the voltages equal to or higher than $150 \mathrm{kV}$, instead, the relative error $C_{\vartheta}$ is always very similar (and even smaller) than $C_{p}$. Additionally, as expected, it is significantly lower than the relative error values obtained by using the 5,200 pilot runs only.

Also in presence of SAs and for the configuration with laterals illustrated in the next Section, results similar to those of Fig. 5 have been obtained.

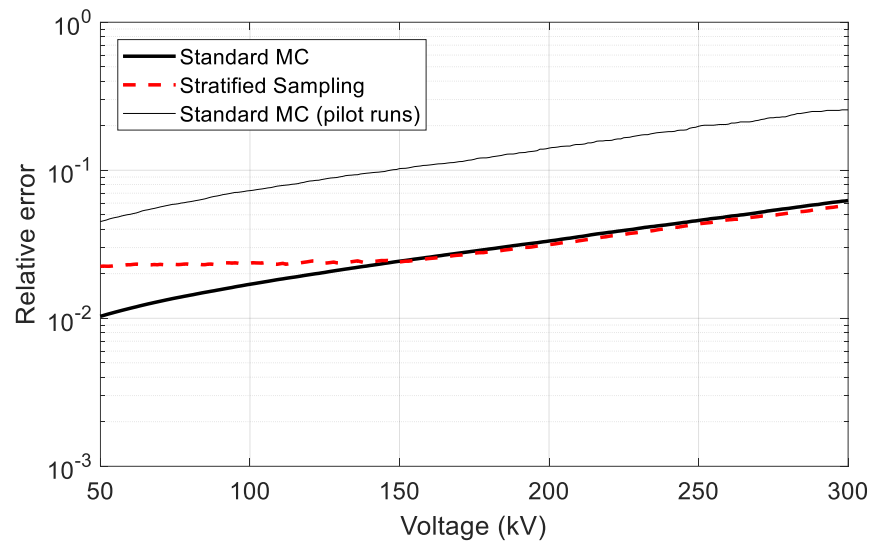

Fig. 5. Comparison of the relative errors of an unprotected straight line calculated by the standard MC (full and limited to the pilot runs only) and the stratified sampling as a function of the withstand voltage.

The proposed stratified sampling method can be easily extended to the case in which the insulation breakdown is represented by means of a specific model, e.g., the disruptive effect criterion [29],[30],[31]). The method is applicable also to more complex coupling models, e.g. the approach proposed in [32], based on the use of the finite element method (FEM) model presented in [33] that allows accounting the shielding and LEMP attenuation effects provided by surrounding buildings.

\section{NETWORK CONFIGURATION}

In this Section, the proposed procedure is applied to the case of a network composed by a main feeder and laterals without SAs. The top-view of the network is reported in Fig. 6. The main feeder is 750-m-long and is matched at the right end. The lines are composed by three overhead conductors of diameter equal to $1 \mathrm{~cm}$, each placed at $9.3 \mathrm{~m}$ above ground. The distances between the external conductors and the central one are equal to $1.5 \mathrm{~m}$ and $0.7 \mathrm{~m}$, respectively.

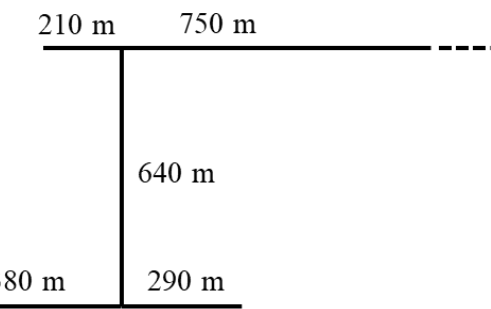

Fig. 6. Configuration of the network

The area considered in the simulation and the location of the lightning events generated by the stratified procedure are shown in Fig. 7. The total area measures approximately 23.8 $\mathrm{km}^{2}$ and the minimum distance between the area borders and the lines is $2 \mathrm{~km}$. The stratified sampling is applied by dividing the area in $m=400$ subdomains, each of area $256.5 \mathrm{~m} \times 232 \mathrm{~m}$. In each subdomain $j$ the number of pilot runs $N_{j}^{s}$ is equal to 25 for a total number of pilot runs equal to 10,000 . The variance $\sigma_{j}^{2}$ is calculated assuming $W=150 \mathrm{kV}$. The stratified sampling procedure is stopped when the relative error $C_{\vartheta}$ reaches the $C_{p}$ value obtained by the standard $\mathrm{MC}$ 
procedure for $150 \mathrm{kV}$ and 100,000 events, which for the network configuration and total area of Fig. 7 is $3.2 \%$. The results are obtained for the same $\mathrm{MC}$ event population generated assuming $W=150 \mathrm{kV}$.

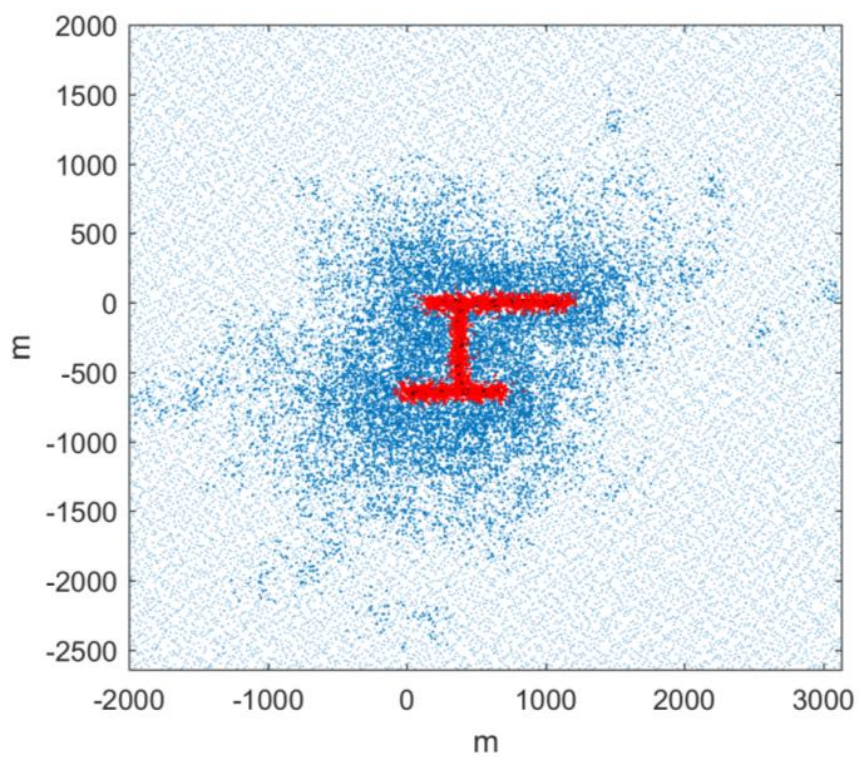

Fig. 7. Position of the events generated by the stratified sampling procedure for the case of a feeder with laterals (direct strikes in red, nearby strikes to ground in blue).

By using the stratified sampling procedure, the $F_{p}$ value at $150 \mathrm{kV}$ is 0.852 whilst by using the standard MC is 0.861 . Fig. 8 shows the agreement between the lightning performances calculated by using the stratified sampling and the standard MC method for all the withstand values between $50 \mathrm{kV}$ and $300 \mathrm{kV}$.

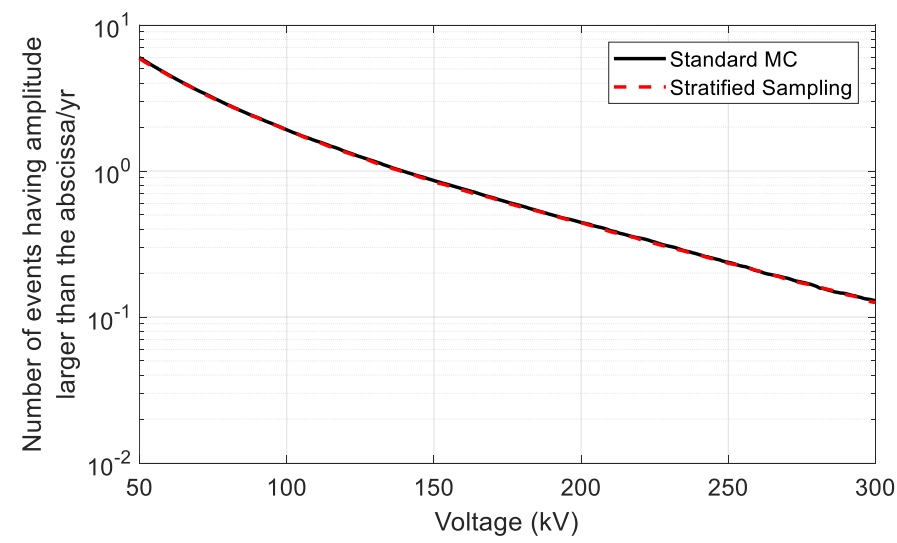

Fig. 8. Comparison of the lightning performance of the network calculated by the standard MC and stratified sampling.

Analogously to Fig. 5 for the case of a single unprotected line, Fig. 9 shows the comparison between $C_{p}$ and $C_{\vartheta}$ values. The $C_{p}$ values are calculated by using the standard MC applied to the full set of 100,000 events and to the set of 10,000 pilot runs used for the initialization of the stratified sampling.

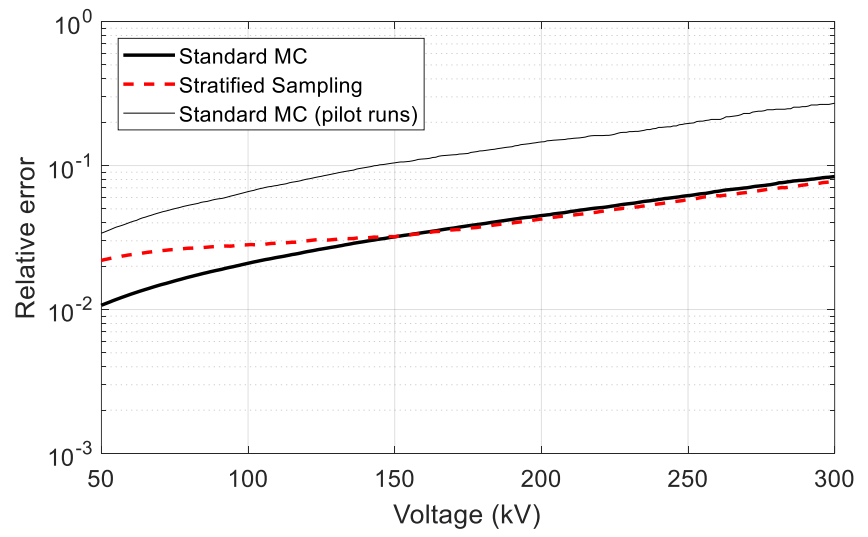

Fig. 9. Comparison of the relative errors calculated by the standard MC (full and limited to the pilot runs only) and the stratified sampling as a function of the withstand voltage.

For the case of the network of Fig. 6, the lightning performance calculation has been carried out also by using the heuristic technique described in [20], which limits the LIOVEMTP calculations only to the events with some probability to induce dangerous voltages, i.e., with amplitudes $V_{\max }$ larger than the considered minimum line-insulation level $\bar{V}$ (equal to $50 \mathrm{kV}$ in Fig. 8). For any event $k$ characterized by $V_{\max , k}<\bar{V}$, the heuristic technique assumes a circular area $\Omega_{k}$ of radius $r_{k}$ centered at the stroke location $P_{k}$. The induced voltages relevant to the $i$-th event are not simulated and directly excluded from the number of dangerous events if event $i$ complies with all the following conditions, namely:

a) $P_{i} \in \Omega_{k}^{*}$ with $V_{\max , k}<\bar{V}$;

b) $I_{i} \leq I_{k}$;

c) $t_{f, i} \geq t_{f, k}$

where $\Omega_{k}^{*}$ defines the points of $\Omega_{k}$ farther than $P_{k}$ from the closest line. For the lightning performance calculation of the network of Fig. $6 r_{k}$ is taken equal to $400 \mathrm{~m}$, that allows to obtain the same $F_{p}$ value of the standard MC with the maximum computational time reduction.

Table II compares the number of MC events relevant to direct strikes and to nearby strikes to ground, the number of required LIOV-EMTP simulations and the computational time reduction with respect to the standard MC.

TABLE II. COMPARISON BETWEEN NUMBER OF EVENTS AND COMPUTATIONAL TIME REDUCTION FOR THE STANDARD MC, THE HEURISTIC MC AND STRATIFIED SAMPLING.

\begin{tabular}{c|c|c|c|c}
\hline \hline Method & $\begin{array}{c}\text { Direct } \\
\text { strikes }\end{array}$ & $\begin{array}{c}\text { Nearby } \\
\text { strikes }\end{array}$ & $\begin{array}{c}\text { LIOV-EMTP } \\
\text { simulations }\end{array}$ & $\begin{array}{c}\text { Time saved } \\
\%\end{array}$ \\
\hline Standard MC & 1,223 & 98,777 & 98,777 & - \\
\hline Heuristic MC & 1,223 & 98,777 & 33,633 & 66 \\
\hline Stratified sampling & 1,742 & 24,858 & 24,858 & 75 \\
\hline \hline
\end{tabular}

In order to analyze the effect of subdomain dimensions, the stratified sampling procedure has been repeated by using a larger number of subdomains, i.e. $m=900$. In this case, each subdomain has area equal to $171 \mathrm{~m} \times 154.7 \mathrm{~m}$ and contains $N_{j}^{s}=25$ pilot runs. The difference with the $F_{p}$ results obtained 
with $m=400$ are not significant, however the number of required LIOV-EMTP simulations increases to 32,348 and the computational time reduction decreases to $67 \%$ (instead of $75 \%)$.

\section{CONCLUSION}

This paper has presented the application of the stratified sampling procedure for the accurate calculation of the lightning performance of power lines and distribution networks with reduced computational time.

With respect to the standard Monte Carlo method presented in the literature, the use of the stratified sampling has several advantages:

- it allows a significant computational time reduction (up to more than $75 \%$ for the cases analyzed in this paper) whilst maintaining the accuracy of the solution;

it reduces the importance of the choice of the smallest area $A$ that includes the perspective stroke locations of all the events inducing voltages exceeding the insulation withstand capability, since the recursive location of the events is governed by the estimation of the conditional variances in the subdomains;

- it is directly applicable to the case of networks with complex configuration, with surge arresters and with the adoption of detailed flashover models, whilst heuristic rules need to be adapted for each specific case.

\section{REFERENCES}

[1] IEEE Std 1410-2010, "IEEE guide for improving the lightning performance of electric power overhead distribution lines," IEEE Std 1410-2010 (Revision IEEE Std 1410-2004), pp. 1-73, 2011.

[2] C. A. Nucci and F. Rachidi, "Interaction of electromagnetic fields generated by lightning with overhead electrical networks," in The Lightning Flash. 2nd Edition, V. Cooray, Ed. IET - Power and Energy Series 69, 2014, pp. 559-610.

[3] P. N. Mikropoulos and T. E. Tsovilis, "Statistical method for the evaluation of the lightning performance of overhead distribution lines," IEEE Trans. Dielectr. Electr. Insul., vol. 20, no. 1, pp. 202-211, Feb. 2013.

[4] A. Borghetti, C. A. Nucci, and M. Paolone, "An Improved Procedure for the Assessment of Overhead Line Indirect Lightning Performance and Its Comparison with the IEEE Std. 1410 Method," IEEE Trans. Power Deliv., vol. 22, no. 1, pp. 684-692, 2007.

[5] CIGRE WG C4.407, "Lightning parameters for engineering applications (TB 549)," Paris, France, 2013.

[6] CIGRE WG C4.404, "Cloud-to-Ground Lightning Parameters derived from Lightning Location Systems. The Effects of System Performance. (TB 376)," Paris, France, 2009.

[7] G. Rubino and B. Tuffin, Rare event simulation using Monte Carlo methods. Wiley, 2009.

[8] J. O. S. Paulino, C. F. Barbosa, I. J. S. Lopes, and W. C. Boaventura, "Assessment and analysis of indirect lightning performance of overhead lines," Electr. Power Syst. Res., vol. 118, pp. 55-61, Jan. 2015.

[9] F. Mahmood, N. A. Sabiha, and M. Lehtonen, "Probabilistic Risk Assessment of MV Insulator Flashover under Combined AC and Lightning-Induced Overvoltages," IEEE Trans. Power Deliv., vol. 30, no. 4, pp. 1880-1888, 2015.

[10] F. Napolitano, A. Borghetti, C. A. Nucci, M. Paolone, F. Rachidi, and J. Mahseredjian, "An advanced interface between the LIOV code and the EMTP-RV," in Proc. 29th International Conference on Lightning Protection (ICLP), Uppsala, Sweden, 2008, pp. 1-12.

[11] F. Napolitano, "An Analytical Formulation of the Electromagnetic Field Generated by Lightning Return Strokes," IEEE Trans. Electromagn. Compat., vol. 53, no. 1, pp. 108-113, Feb. 2011.
[12] M. Paolone, F. Rachid, A. Borghetti, C. A. Nucci, M. Rubinstein, V. A. Rakov, and M. A. Uman, "Lightning electromagnetic field coupling to overhead lines: Theory, numerical simulations, and experimental validation," IEEE Trans. Electromagn. Compat., vol. 51, no. 3, pp. 532547,2009

[13] F. Napolitano, A. Borghetti, M. Paolone, and M. Bernardi, "Voltage transient measurements in a distribution network correlated with data from lightning location system and from sequence of events recorders," Electr. Power Syst. Res., vol. 81, no. 2, pp. 237-253, 2011.

[14] J. Chen and M. Zhu, "Calculation of Lightning Flashover Rates of Overhead Distribution Lines Considering Direct and Indirect Strokes," IEEE Trans. Electromagn. Compat., vol. 56, no. 3, pp. 668-674, Jun. 2014.

[15] E. Soto, D. Del Río, E. Pérez, and C. Younes, "Lightning performance of overhead distribution network assessment using fuzzy ground flash density concept," in 2008 IEEE/PES Transmission and Distribution Conference and Exposition: Latin America, T and D-LA, 2008, pp. 1-6.

[16] M. Brignone, F. Delfino, R. Procopio, M. Rossi, and F. Rachidi, "Evaluation of Power System Lightning Performance - Part II: Application to an Overhead Distribution Network," IEEE Trans. Electromagn. Compat., vol. 59, no. 1, pp. 146-153, 2017.

[17] F. Napolitano, M. Paolone, A. Borghetti, C. A. Nucci, F. Rachidi, V. A. Rakov, J. Schoene, and M. A. Uman, "Interaction between grounding systems and nearby lightning for the calculation of overvoltages in overhead distribution lines," in Proc. 2011 IEEE PES PowerTech, Trondheim, Norway, 2011.

[18] S. Chen, Y. Zhang, C. Chen, X. Yan, W. Lu, and Y. Zhang, "Influence of the ground potential rise on the residual voltage of low-voltage surge protective devices due to nearby lightning flashes," IEEE Trans. Power Deliv., vol. 31, no. 2, pp. 596-604, 2016.

[19] I. Bendato, M. Brignone, F. Delfino, R. Procopio, and F. Rachidi, “A Methodology to Reduce the Computational Effort in the Evaluation of the Lightning Performance of Distribution Networks," Atmosphere (Basel)., vol. 7, no. 11, p. 147, 2016.

[20] A. Borghetti, C. A. Nucci, and M. Paolone, "Indirect-Lightning Performance of Overhead Distribution Networks With Complex Topology," IEEE Trans. Power Deliv., vol. 24, no. 4, pp. 2206-2213, Oct. 2009.

[21] A. Borghetti, G. J. G. Dos Santos, D. R. Fagundes, G. P. Lopes, M. L. B. Martinez, F. Napolitano, C. A. Nucci, F. Tossani, D. R. Fagundes, G. P. Lopes, and M. L. B. Martinez, "Indirect Lightning Performance of a Real Distribution Network With Focus on Transformer Protection," in 32nd International Conference on Lightning Protection, Shanghai, China, 2014.

[22] F. Napolitano, F. Tossani, A. Borghetti, C. A. Nucci, M. L. B. Martinez, G. P. Lopes, G. J. G. Dos Santos, and D. R. Fagundes, "Lightning performance of a real distribution network with focus on transformer protection," Electr. Power Syst. Res., vol. 139, pp. 60-67, 2016.

[23] P. Brandimarte, Handbook in Monte Carlo Simulation: Applications in Financial Engineering, Risk Management, and Economics. Wiley, 2014.

[24] E. Zio, The Monte Carlo Simulation Method for System Reliability and Risk Analysis. Springer Publishing Company, Incorporated, 2014.

[25] A. Borghetti, F. Napolitano, C. A. Nucci, and F. Tossani, "Influence of the return stroke current waveform on the lightning performance of distribution lines," IEEE Trans. Power Deliv., vol. 32, no. 4, pp. 1800$1808,2017$.

[26] Cigré Working Group 33.01, "Guide to procedures for estimating the lightning performance of transmission lines (TB 63)," CIGRE, Paris, 1991.

[27] C. A. Nucci, F. Rachidi, M. Ianoz, and C. Mazzetti, "Lightning-Induced Voltages on Overhead Lines," IEEE Trans. Electromagn. Compat., vol. 35, no. 1, pp. 75-86, 1993.

[28] F. Tossani, F. Napolitano, A. Borghetti, C. A. Nucci, G. P. Lopes, M. L. B. Martinez, and G. J. G. Dos Santos, "Estimation of the Influence of direct strokes on the lightning performance of overhead distribution lines," in 2015 IEEE PowerTech Conference, Eindhoven, 2015.

[29] M. Darveniza and A. E. Vlastos, "The generalized integration method for predicting impulse volt-time characteristics for non-standard wave shapes-a theoretical basis," IEEE Trans. Electr. Insul., vol. 23, no. 3, pp. 373-381, 1988.

[30] A. De Conti, E. Perez, E. Soto, F. H. Silveira, S. S. Visacro, and H. Torres, "Calculation of Lightning-Induced Voltages on Overhead Distribution Lines Including Insulation Breakdown," IEEE Trans. Power Deliv., vol. 25, no. 4, pp. 3078-3084, Oct. 2010.

[31] A. Borghetti, F. Napolitano, C. A. Nucci, and F. Tossani, "Response of 
distribution networks to direct and indirect lightning: Influence of surge arresters location, flashover occurrence and environmental shielding," Electr. Power Syst. Res., vol. 153, pp. 73-81, 2017.

[32] F. Tossani, A. Borghetti, F. Napolitano, A. Piantini, and C. A. Nucci, "Lightning Performance of Overhead Power Distribution Lines in Urban Areas," IEEE Trans. Power Deliv., vol. pp, no. 99, p. in press, 2017.

[33] A. Borghetti, F. Napolitano, C. A. Nucci, and M. Paolone, "Effects of nearby buildings on lightning induced voltages on overhead power distribution lines," Electr. Power Syst. Res., vol. 94, pp. 38-45, 2013.

Fabio Napolitano (M'16-SM'17) is assistant professor at the Department of Electrical, Electronic and Information Engineering of the University of Bologna, Italy. From the same university, he received the M.S. degree (with hons.) in electrical engineering in 2003 and the Ph.D. degree in electrical engineering in 2009. Since his graduation he collaborated with the Power Systems group of the University of Bologna on the analysis of power systems transients, in particular those due to indirect lightning strokes, and lightning protection systems.

Fabio Tossani (S'15-M'16) was born in Bologna, Italy, in 1988. He received the B.S. (Hons.), M.S. (Hons.) and Ph.D. degree in electrical engineering from the University of Bologna, Italy, in 2010, 2012 and 2016, respectively. He is currently a research fellow of the Power Systems group of the University of Bologna. His research interests are power system transients, with particular reference to lightning electromagnetic pulse interaction with electrical networks, power systems protection and the integration of renewables in power distribution networks.

Alberto Borghetti (M'97-SM'03-F'15) graduated (with honors) in electrical engineering from the University of Bologna, Italy, in 1992. Since then he has been working with the power system group of the same University, where he is now Associate Professor. His research and teaching activities are in the areas of power system analysis, power system restoration after blackout, electromagnetic transients, optimal generation scheduling, and distribution system operation. He has been granted the ICLP Scientific Committee Award 2016. From 2010 to 2016 he has served as an Editor of IEEE Trans. on Smart Grid and he is an editorial board member of Electric Power Systems Research.

Carlo Alberto Nucci (M'91-SM'02-F'07) is a full professor and the head of the Power Systems Laboratory of the Department of Electrical, Electronic and Information Engineering 'Guglielmo Marconi,' University of Bologna, Bologna, Italy. $\mathrm{He}$ is author or coauthor of over 300 scientific papers published on peer- reviewed journals or on proceedings of international conferences, of six book chapters edited by IEE, IET (two), Kluwer, Rumanian Academy of Science and WIT press and of a couple of IEEE Standards and some CIGRE technical brochures. He is a Fellow of the IET, and of CIGRE, of which he is also honorary member, and has received some best paper/technical international awards, including the CIGRE Technical Committee Award and the ICLP Golde Award. From January 2006 to September 2012, he has served as a Chairman of Cigre Study Committee C4 'System Technical Performance.' Since January 2010, he has been an Editorin-Chief of the Electric Power Systems Research journal, Elsevier. He has served as IEEE PES Region 8 Rep in the years 2009 and 2010. He is doctor honoris causa of the University Politehnica of Bucharest and corresponding member of the Bologna Science Academy. He has served as the President of the Italian Group of University Professors of Electrical Power Systems (GUSEE) from 2012 to 2015. He is an Advisor of the Global Resource Management Program of Doshisha University, Kyoto, supported by the Japanese Ministry of Education and Science. He has been serving as member of the EU Smart City Stakeholder Platform since 2013, and since 2014 is representing PES in the IEEE Smart City Initiatives Program. 